\title{
Sensorless Passivity Based Control of a DC Motor via a Solar Powered Sepic Converter-Full Bridge Combination
}

\author{
Jesús Linares-Flores ${ }^{\dagger}$, Hebertt Sira-Ramírez* , Edel F. Cuevas-López ${ }^{* *}$, and Marco A. Contreras-Ordaz ${ }^{* *}$ \\ †** Instituto de Electrónica y Mecatrónica, Universidad Tecnológica de la Mixteca, Huajuapan de León, Oaxaca, México \\ ${ }^{*}$ Centro de Investigación y de Estudios Avanzados del IPN CINVESTAV, D. F. México, México
}

\begin{abstract}
This article deals with the sensor-less control of a DC Motor via a SEPIC Converter-Full Bridge combination powered through solar panels. We simultaneously regulate, both, the output voltage of the SEPIC-converter to a value larger than the solar panel output voltage, and the shaft angular velocity, in any of the turning senses, so that it tracks a pre-specified constant reference. The main result of our proposed control scheme is an efficient linear controller obtained via Lyapunov. This controller is based on measurements of the converter currents and voltages, and the DC motor armature current. The control law is derived using an exact stabilization error dynamics model, from which a static linear passive feedback control law is derived. All values of the constant references are parameterized in terms of the equilibrium point of the multivariable system: the SEPIC converter desired output voltage, the solar panel output voltage at its Maximun Power Point (MPP), and the DC motor desired constant angular velocity. The switched control realization of the designed average continuous feedback control law is accomplished by means of a, discrete-valued, Pulse Width Modulation (PWM). Experimental results are presented demonstrating the viability of our proposal.
\end{abstract}

Key Words: Passivity Based Control, Sensorless Control, SEPIC-Full Bridge Converter, Solar Cells

\section{INTRODUCTION}

Solar photovoltaic energy is one of the most popular renewable energy technologies since it uses the energy radiation that we receive from the sun which is available practically everywhere. Solar photovoltaic energy is mainly used as an alternative energy source for residential or industrial electrical systems [1]. However, another important application is the use of solar energy in transportation. The better known applications are referred to the hybrid solar vehicles which are frequently mentioned as an alternative to conventional combustion engine equipped vehicles [2]. Other interesting application of electric vehicles is constituted by the "space elevator" concept, which proposes to use a carbon nanotube cable, connected between the earth and a counterweight in space, to allow a vehicle to travel to space and back. The vehicle moves on the cable using electric motors and the power is produced from solar panels that are illuminated by a ground based laser [3].

On the other hand, the combination of DC-to-DC power

Manuscript received Nov. 4, 2010; revised May 13, 2011

Recommended for publication by Associate Editor Jun-Keun Ji.

†Corresponding Author: jlinares@mixteco.utm.mx

Tel: +52-953-5320214(Ext.760), Universidad Tecnológica de la Mixteca

* Centro de Investigación y de Estudios Avanzados del IPN CINVESTAV, México

** Instituto de Electrónica y Mecatrónica, Universidad Tecnológica de la Mixteca, México converters, powered through a solar panel, with DC motor drives is a new alternative to develop motion systems that do not need conventional electrical energy for being operated. This type of solar drive we can find in the work reported in [4]. In this application, the power converters transfer the solar panel power to the load represented by a DC motor [3], [5]. The output voltage of the power converter delivers the required voltage supply to the DC-motor in accordance with the demanded task, represented, for example, by the achievement of the desired angular velocity profile or of the desired angular position reference trajectory [7], [11]. Nonlinear average models replacing the discrete nature of the switching control in the transistor are commonly used in the feedback control design for DC motor/dc-to-dc power converters. Following the design of these models, speed sensorless controllers were designed [6], [10], [11]. Switched implementations of average dynamic output feedback control laws; by means of a PWM-modulator, are widely known in classic communications and analog signal encoding literature; for novel applications see [8], [9]. The DC motor/SEPIC converter combination powered via a solar panel, has been used for the speed sensorless control. However, until now, the controller designed can only regulate the angular velocity shaft on positive direction [5]. Likewise, the DC motor/SEPIC converter combination powered via an AC-toDC converter, has been used for a direct regulation of the motor shaft speed on positive direction [12]. While, the SEPIC 


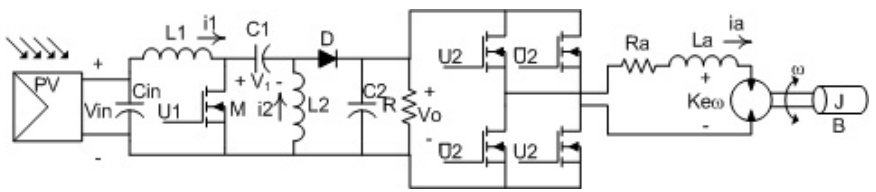

Fig. 1. Model of a cascaded SEPIC-Full Bridge converter/dc-motor combination powered via a solar panel.

converter topology has been widely used in control methods for tracking maximum power point in photovoltaic power systems [13]-[15].

In this article, we develop a solar panel fed drive for DC motor speed regulation. The power converters (SEPICFull Bridge) are used as an interface on which we can, simultaneously, control the converter's output voltage and the angular speed of the DC motor, under certain conditions of solar radiation. The controller drives the combined system to a constant set-point allowing the output voltage of the SEPIC converter feed the load represented by a second Full-Bridge converter feeding, in turn, a DC motor. The angular velocity of the DC-motor, driven by the Full-Bridge converter, is therefore also regulated. We address the cascade arrangement of a SEPIC DC-to-DC power converter and the Full Bridge converter, a "SFB-converter". The main task is to achieve the regulation of the angular velocity to a constant value in both possible directions of rotation of the DC-motor shaft. The regulation is carried under a certain maximum power condition on the solar radiation hitting the solar panel. A simple linear time varying state feedback controller, based on exact static error dynamics passive output feedback, is shown to globally stabilize the state trajectory regulation error to zero while requiring only the measurement of the converter currents and voltages, and the DC motor armature current for the synthesis of the feedback law. The dynamic average multivariable model of the "SFB converter/DC-motor" combination is shown to conform to a special energy managing structure which is suitable for passivity-based feedback techniques. The reference signals of the multi-variable system are generated via the nominal equilibrium values of the composite plant. We use a desired constant value of the SEPIC converter output voltage, we assume that the solar panel output voltage operated at the Maximum Power Point (MPP), and we set a desired constant value for the DC motor shaft angular velocity. These desired constant references, in turn, completely define the reference constants of the input currents, output voltages, and average controls of the "SFB-converter". The desired constant references can all be computed in an off-line fashion.

This article is organized as follows: Section II deals with the average model of the multi-variable system "SFBconverter/DC-motor", including the converters, in a cascaded arrangement, being powered via a solar panel. Section III is concerned with the desired constant references generation required by the proposed linear time feedback controller. Section IV presents a linear feedback controller derived on the basis of elementary passivity considerations. We term such a scheme "the Exact Static Error Dynamics Passive Output Feedback" controller (ESEDPOF), see [11], [16]. In Section $\mathrm{V}$, the experimental setup is described and used for validating the performance of the proposed control policy via actual laboratory experiments. The last section presents some conclusions and perspectives for further work.

\section{The Average Model of the Multi-VAriable SYSTEM "SFB-CONVERTER/DCMOTOR" POWERED VIA A SOLAR PANEL}

We consider a combination of a SEPIC DC-to-DC power converter cascaded by a Full Bridge, both powered by a solar panel and loaded by a DC-motor, as depicted in Fig. 1. Using Kirchhoff's current and voltage laws and Newton's second law of mechanics, we obtain the following average model of the multivariable system:

$$
\begin{aligned}
L_{1} \frac{d i_{L_{1}}}{d t} & =v_{i n}(t)-\left(1-u_{1_{a v}}\right)\left(v_{1}+v_{0}\right) \\
L_{2} \frac{d i_{L_{2}}}{d t} & =v_{1} u_{1_{a v}}-\left(1-u_{1_{a v}}\right) v_{0} \\
C_{1} \frac{d v_{1}}{d t} & =-i_{L_{2}} u_{1_{a v}}+\left(1-u_{1_{a v}}\right) i_{L_{1}} \\
C_{2} \frac{d v_{0}}{d t} & =-\frac{v_{0}}{R}+\left(1-u_{1_{a v}}\right)\left(i_{L_{1}}+i_{L_{2}}\right)-i_{a} u_{2_{a v}} \\
L_{a} \frac{d i_{a}}{d t} & =-R_{a} i_{a}-K_{e} \omega+v_{0} u_{2_{a v}} \\
J \frac{d \omega}{d t} & =K_{t} i_{a}-B \omega-\tau_{L}
\end{aligned}
$$

where the variables in the model represent:

$v_{\text {in }}$ solar panel output voltage supply;

$i_{L_{1}}$ inductor current in the SEPIC converter $\left(L_{1}\right)$;

$i_{L_{2}}$ inductor current in the SEPIC converter $\left(L_{2}\right)$;

$v_{1} \quad$ Capacitor voltage in the SEPIC converter $\left(C_{1}\right)$;

$v_{0} \quad$ Capacitor voltage in the SEPIC converter $\left(C_{2}\right)$;

$i_{a}$ armature current;

$\omega$ angular velocity of the motor shaft;

$u_{1_{a v}} \quad$ average control input of the SEPIC converter;

$u_{2_{a v}} \quad$ average control input of the Full Bridge converter;

$\tau_{L} \quad$ load torque;

Using matrix notation, the nonlinear system (1)-(6) may be represented in the following energy-management, or passive, form:

$$
\mathbf{A} \dot{x}=\left[\mathbf{J}\left(u_{a v}\right)-\mathbf{R}\right] x+\mathbf{B} u_{a v}+\eta(t)
$$

where $x$ is an 6 -dimensional vector, $\mathbf{A}$ is a symmetric, positive definite, constant, matrix, $\mathbf{J}\left(u_{a v}\right)$ is a skew symmetric matrix, for all $u_{a v}$, of the form

$$
\mathbf{J}\left(u_{a v}\right)=\mathbf{J}_{0}+\sum_{i=1}^{m} \mathbf{J}_{i} u_{i, a v} .
$$

Here, the matrices $\mathbf{J}_{i}, i=0,1, \ldots, m$ are constant skew symmetric matrices. $\mathbf{R}$ is a symmetric, positive semi-definite constant matrix. $\mathbf{B}$ is a constant $6 \times 2$ matrix. The vector $u_{a v}$ is the average control input vector assumed to be 2dimensional, with its two components, $u_{i, a v}, i=1,2$ taking values, respectively, in the closed sets: $[0,1]$ and $[-1,1]$ of the real line. The vector, $\eta(t)$, is an 6-dimensional smooth vector function of time, $t$, or, in some instances, it represents a vector of constant entries. Note that the vector $\mathbf{R} x$ represents the dissipative field of the system while $\mathbf{J}\left(u_{a v}\right) x$ represents the, control input dependent, conservative field of the system. 
The control input channels are represented by the columns of the constant matrix $\mathbf{B}$ while $\eta(t)$ represent the external inputs to the system, which is constituted, generally speaking, by a either a battery voltage value, a solar panel output voltage, or an ac line voltage. This vector also contains the external disturbance torque input $\tau$.

Specifically, in attention to equation (7), we have :

$$
x^{T}=\left(i_{L_{1}}, i_{L_{2}}, v_{1}, v_{0}, i_{a}, \omega\right)
$$

with

$$
\mathbf{A}=\operatorname{diag}\left(L_{1}, L_{2}, C_{1}, C_{2}, L_{a}, J\right)
$$

while the matrices in (7) and (8) are given by

$$
\begin{aligned}
& \mathbf{J}\left(u_{a v}\right)=\left(\begin{array}{ccc}
0 & 0 & u_{1_{a v}}-1 \\
0 & 0 & u_{1_{a v}} \\
1-u_{1_{a v}} & -u_{1_{a v}} & 0 \\
1-u_{1_{a v}} & 1-u_{1_{a v}} & 0 \\
0 & 0 & 0 \\
0 & 0 & 0
\end{array}\right. \\
& \left.\begin{array}{ccc}
u_{1_{a v}}-1 & 0 & 0 \\
u_{1_{a v}}-1 & 0 & 0 \\
0 & 0 & 0 \\
0 & -u_{2_{a v}} & 0 \\
u_{2_{a v}} & 0 & -K_{e} \\
0 & K_{t} & 0
\end{array}\right) .
\end{aligned}
$$

Note that for arbitrary values of $u_{1_{a v}}, u_{2_{a v}}$, the matrix $\mathbf{J}\left(u_{a v}\right)$ is skew-symmetric, that is $\mathbf{J}^{T}\left(u_{a v}\right)=-\mathbf{J}\left(u_{a v}\right)$. Since, $K_{e}=K_{t}$, due to energy conservation (i.e., the power absorbed by the back electro-motive-force (emf) is fully converted into mechanical power, see [7]). The matrix, $\mathbf{R}$, is symmetric and positive-semidefinite, i.e., $\mathbf{R}^{T}=\mathbf{R} \geq \mathbf{0}$.

\section{A. Coupling of the solar panel and the SEPIC converter}

Using the Linear Reoriented Coordinates Method, discussed in [17], we have the optimum voltage given by:

$$
V_{o p}=V_{o c}+b V_{o c} \ln \left[b-b e^{-\left(\frac{1}{b}\right)}\right]
$$

While that the maximum power of the PV panel is then given by:

$$
P_{\max }=V_{o p} I_{o p}=V_{o p} \frac{I_{s c}-I_{s c} e^{\left(\frac{V_{o p}}{b V_{o c}}-\frac{1}{b}\right)}}{1-e^{-\left(\frac{1}{b}\right)}}
$$

From (13), we find that the PV panel characteristic constant, $b$, is given by:

$$
b=\frac{\frac{V_{o p}}{V_{o c}}-1}{\ln \left[1-\frac{I_{o p}}{I_{s c}}\right]}
$$

One way to find the constant, $b$, of our solar panel is by using the information given by the Solarex manufacturers data sheet, [19], using the method presented in [18] where,

$$
b=\frac{\frac{16.8 V}{21 V}-1}{\ln \left[1-\frac{2.97 A}{3.23 A}\right]} \approx 0.08
$$

The value $b$ in (15) is used throughout in the developments concerning the experimental setup.

There exists a direct relation between the PV panel characteristic constant, $b$, the fill factor, $f f$, and the efficiency of the PV panel, $\left(\eta_{p}\right)$,

$$
\begin{aligned}
& f f=\frac{P_{\max }}{I_{s c} V_{o c}}=\frac{V_{o p}\left(1-e\left(\frac{V_{o p}}{b V_{o c}}-\frac{1}{b}\right)\right)}{V_{o c}\left(1-e^{-\left(\frac{1}{b}\right)}\right)} \\
& \eta_{p}=\frac{V_{o p} I_{o p}}{P_{i n}}
\end{aligned}
$$

where $P_{i n}$ is input light power. The $\mathrm{I}-\mathrm{V}$ and $\mathrm{P}-\mathrm{V}$ characteristic curves for a percentage of effective intensity of light over the SX50U solar panel are showed in data sheet [19].

At the point of maximum power the photovoltaic panel has a dual behavior; it may function as a current source, or as a voltage source. For the panel to function as a voltage source it needs to have a capacitor, of appropriate value, connected between its output terminals. This ensures that the PV panel works as a DC voltage source, which acts as the main power source for the SEPIC converter. This coupling capacitor significantly reduces the output voltage variations of the panel. Figure 1 shows the link between the PV panel and the SEPIC converter via the capacitor. Considering that all the energy of the current variation is supplied by the capacitor, $C_{i n}$, connected in parallel with the panel, the following equation is obtained (see [1]).

$$
\frac{1}{2} C_{i n}\left(V_{\max }^{2}-V_{\min }^{2}\right)=\frac{1}{2} L_{1}\left(I_{\max }^{2}-I_{\min }^{2}\right)
$$

Solving for the $C_{i n}$ parameter from (16), we obtain,

$$
\begin{aligned}
C_{i n} & =\frac{L_{1}\left(I_{\max }^{2}-I_{\min }^{2}\right)}{\left(V_{\max }^{2}-V_{\min }^{2}\right)} \\
& =\frac{L_{1}\left(I_{\max }+I_{\min }\right) \Delta I}{\left(V_{\max }+V_{\min }\right) \Delta V} \\
& =\frac{L_{1} \cdot I_{\mathrm{nom}} \cdot \Delta I}{V_{\mathrm{nom}} \cdot \Delta V}
\end{aligned}
$$

where:

$V_{\text {nom }}=2\left(V_{\max }+V_{\min }\right)$ nominal voltage in the SEPIC converter input.

$I_{\text {nom }}=2\left(I_{\max }+I_{\min }\right)$ nominal current in the SEPIC converter input.

$\Delta V$ is the ripple voltage in the SEPIC converter input.

$\Delta I$ is the ripple current in the SEPIC converter input.

The value of the coupling capacitor, $C_{i n}$, depends on the design of the maximum and minimum input currents to the SEPIC converter. It also depends on the nominal values of voltage and current flowing out of photovoltaic panel as well as the value of the SEPIC converter input inductor $L_{1}$. Therefore, using the values of the designed SEPIC converter and the manufacturer's data sheet for the PV panel, we can compute the appropriate value of the coupling capacitor to use the PV panel as a DC voltage source. 


\section{Reference Variables of the Multi-Variable SYSTEM}

Based on the given average model equations, (1) to (6), we define the reference model, to regulate the system in an operating point, through the following nominal equations

$$
\begin{aligned}
L_{1} \frac{d \bar{\imath}_{L_{1}}}{d t} & =\underbrace{\bar{v}_{i n}(t)}_{:=V_{o p}}-\left(1-\bar{u}_{1_{a v}}\right)\left(\bar{v}_{1}+\bar{v}_{0}\right) \\
L_{2} \frac{d \bar{l}_{L_{2}}}{d t} & =\bar{v}_{1} u_{1_{a v}}-\left(1-\bar{u}_{1_{a v}}\right) \bar{v}_{0} \\
C_{1} \frac{d \bar{v}_{1}}{d t} & =-\bar{\imath}_{L_{2}} u_{1_{a v}}+\left(1-\bar{u}_{1_{a v}}\right) \bar{\imath}_{L_{1}} \\
C_{2} \frac{d \bar{v}_{0}}{d t} & =-\frac{\bar{v}_{0}}{R}+\left(1-\bar{u}_{1_{a v}}\right)\left(\bar{\imath}_{L_{1}}+\bar{\imath}_{L_{2}}\right)-\bar{\imath}_{a} \bar{u}_{2_{a v}} \\
L_{a} \frac{d \bar{\imath}_{a}}{d t} & =-R_{a} \bar{\imath}_{a}-K_{e} \bar{\omega}+\bar{v}_{0} \bar{u}_{2_{a v}} \\
J \frac{d \bar{\omega}}{d t} & =K_{t} \bar{\imath}_{a}-B \bar{\omega}-\bar{\tau}_{L}
\end{aligned}
$$

Putting the reference model in its passive form, we have

$$
\mathbf{A} \dot{\bar{x}}=\left[\mathbf{J}\left(\bar{u}_{a v}\right)-\mathbf{R}\right] \bar{x}+\bar{\eta}(t)
$$

Equating the right side of equation (19) to zero, and substituting the two desired constant voltages of the SEPIC converter as well as the desired constant angular velocity, we have the following equilibrium point:

$$
\begin{aligned}
\bar{v}_{1} & =\bar{v}_{i n}(t)=V_{o p} \\
\bar{v}_{0} & =V_{d} \\
\bar{\omega} & =\omega_{d} \\
\bar{\imath}_{a} & =\frac{B}{K} \omega_{d} \\
\bar{u}_{1_{a v}} & =\frac{V_{d}}{V_{o p}+V_{d}} \\
\bar{u}_{2_{a v}} & =\frac{\omega_{d}\left(\frac{B R_{a}}{K}+K\right)}{V_{d}} \\
\bar{\imath}_{L_{1}} & =\frac{V_{d}^{2}}{R V_{o p}}+\frac{\left(R_{a} B^{2}+K^{2} B\right) \omega_{d}^{2}}{V_{o p} K^{2}} \\
\bar{\imath}_{L_{2}} & =\frac{V_{d}}{R_{L}}+\frac{\left(R_{a} B^{2}+K^{2} B\right) \omega_{d}^{2}}{V_{d} K^{2}} \\
\bar{\tau}_{L} & =0
\end{aligned}
$$

The vector of reference states is based on the nominal values pre-specified by equations (20), (21) and (22). Thus, we define the vector of state references as

$$
\bar{x}^{T}=\left(\bar{\imath}_{L_{1}}, \bar{\imath}_{L_{2}}, \bar{v}_{1}, \bar{v}_{0}, \bar{\imath}_{a}, \bar{\omega}\right)
$$

Note that the reference value of the voltage $v_{1}$ is the optimum voltage of the solar panel (see (12)). Thus, the controller $u_{1_{a v}}$ will regulate the capacitor voltage in the SEPIC converter $\left(C_{1}\right)$ to the optimum voltage of the solar panel whenever the solar irradiance is suitable (see (20)). The main objective of the control is to hold, at all times, the SEPIC output voltage $\left(v_{0}\right)$ to desired reference $\left(V_{d}\right)$, which is maximum nominal value of the DC motor supply. This is enforced with the purpose of controlling the angular velocity of the DC motor, from a zero speed, to the a maximum nominal speed marked on the data sheet provided by the DC motor manufacturer.

Nominal reference values developed in (20) to (28) will be used by the multivariable controller synthesized on elementary passivity considerations. Here we use the Exact Static Error Dynamics Passive Outputs Feedback (ESEDPOF) which is the stabilization counterpart of the Exact Tracking Error Dynamics Passive Output Feedback (ETEDPOF) introduced in [16].

\section{Passivity-Based Average Multivariable CONTROLler DeSign}

It is desired to control each of the output variables to their reference values. The first output to be regulated is the SEPIC converter output voltage, $v_{0}$. The second output is the angular velocity of the DC-motor, $\omega$. The reference values for these variables have a direct dependence on the solar panel output voltage. Therefore, according to the prevailing solar radiation, we can maneuver the average controller, $u_{1 a v}$ to achieve a desired value $\left(V_{d}\right)$ for the SEPIC output voltage $\left(v_{0}\right)$. This desired value is the input supply source of the full bridge converter, where $V_{d}$ is also the maximum nominal value of the DC motor. By means of $u_{2, a v}$ we can regulate the DC motor angular velocity, from zero to its maximum velocity. Computing the stabilization error dynamics from the subtraction of (7) with (19), we have

$$
\begin{aligned}
\mathbf{A} \dot{x}-\mathbf{A} \dot{\bar{x}}= & \left(\mathbf{J}\left(u_{a v}\right)-\mathbf{R}\right) x-\left(\mathbf{J}\left(\bar{u}_{a v}\right)-\mathbf{R}\right) \bar{x} \\
& +(\eta-\bar{\eta}) \\
\mathbf{A} \dot{e}= & \left(\mathbf{J}\left(u_{a v}\right)-\mathbf{R}\right) x-\left(\mathbf{J}\left(\bar{u}_{a v}\right)-\mathbf{R}\right) \bar{x} \\
& +\underbrace{(\eta-\bar{\eta})}_{:=\vartheta}
\end{aligned}
$$

Considering that $\vartheta=0$, due in part to $\tau_{L}=\bar{\tau}_{L}=0$ and $v_{\text {in }}(t)=\bar{v}_{\text {in }}(t)=V_{o p}$, after some algebraic manipulations carried out on the error dynamics, while denoting the average control input error as: $e_{u_{a v}}=u_{a v}-\bar{u}_{a v}$, we have

$$
\mathbf{A} \dot{e}=\mathbf{J}\left(u_{a v}\right) e+\left(\mathbf{J}\left(u_{a v}\right)-\mathbf{J}\left(\bar{u}_{a v}\right)\right) \bar{x}-\mathbf{R} e
$$

Due to the linearity of equation (30) the expression $\mathbf{J}\left(u_{a v}\right)-$ $\mathbf{J}\left(\bar{u}_{a v}\right)$ may be written as,

$$
\mathbf{J}\left(u_{a v}\right)-\mathbf{J}\left(\bar{u}_{a v}\right)=\underbrace{\left.\frac{\partial \mathbf{J}\left(u_{a v}\right)}{\partial u_{a v}}\right|_{u_{a v}=\bar{u}_{a v}}}_{=: \mathbf{J}} e_{u_{a v}}
$$

Therefore, equation (8) is written as

$$
\sum_{i=1}^{m} \mathbf{J}_{i}\left(u_{i, a v}-\bar{u}_{i, a v}\right)=\sum_{i=1}^{m} \mathbf{J}_{i} \mathbf{e}_{i, u_{a v}}
$$

Substitution of equation (31) in (30) results in

$$
\begin{aligned}
\mathbf{A} \dot{e} & =\mathbf{J}\left(u_{a v}\right) e-\mathbf{R} e+\sum_{i=1}^{m} \mathbf{J}_{i} \mathbf{e}_{i, u_{a v}} \bar{x} \\
& =\mathbf{J}\left(u_{a v}\right) e-\mathbf{R} e+\underbrace{\left[\left(\mathbf{J}_{1} \bar{x}, \ldots, \mathbf{J}_{m} \bar{x}\right)\right]}_{=: \overline{\mathbf{B}}} \mathbf{e}_{i, u_{a v}} \\
& =\mathbf{J}\left(u_{a v}\right) e-\mathbf{R} e+\overline{\mathbf{B}} \mathbf{e}_{u_{a v}}
\end{aligned}
$$


We address this last expression ad the the open loop stabilization error dynamics.

We choose the Lyapunov function candidate,

$$
V(e)=\frac{1}{2} e^{T} A e
$$

The symmetry of the matrix A and the antisymmetry of the matrix $\mathbf{J}\left(u_{a v}\right)$, for any $u_{a v}$, then implies that

$$
\dot{V}(e)=e^{T} A e=-e^{T} \mathbf{R} e+e^{T} \overline{\mathbf{B}} \mathbf{e}_{u_{a v}}
$$

Let $\Gamma$ be a positive definite constant matrix. The control input error, $u_{a v}$, in (34) may be conveniently specified to be

$$
\mathbf{e}_{u_{a v}}=u_{a v}-\bar{u}_{a v}=-\Gamma \overline{\mathbf{B}}^{T} e
$$

The equation given by (35) is a linear stabilization output error feedback controller. We address this controller as the "Exact Static Error Dynamics Passive Output Feedback" or, shortly, ESEDPOF controller, see [9], [11], [16]. It follows that

$$
\begin{aligned}
\dot{V}(e) & =-e^{T} \mathbf{R} e-e^{T} \overline{\mathbf{B}} \boldsymbol{\Gamma} \overline{\mathbf{B}}^{T} e \\
& =-e^{T} \underbrace{\left(\mathbf{R}+\overline{\mathbf{B}} \boldsymbol{\Gamma} \overline{\mathbf{B}}^{T}\right)}_{=: \tilde{\mathbf{R}}} e \leq 0
\end{aligned}
$$

When the matrix, $\tilde{\mathbf{R}}$, from (36), is positive definite, we say that the dissipation matching condition is being satisfied. If the matrix term, $\tilde{\mathbf{R}}$ is only positive semi-definite, we say we have a defect on the dissipation matching condition for the fed back system. Roughly speaking, this condition states that if the natural dissipation of the system is not fully present in the entire state space, full dissipation may be still achieved by an appropriate, supplementary, feedback control action.

In our case, for any constant, positive definite, symmetric matrix $\Gamma$ we find that the matrix $\tilde{\mathbf{R}}$ is given by:

$$
\tilde{\mathbf{R}}=\mathbf{R}+\overline{\mathbf{B}} \boldsymbol{\Gamma} \overline{\mathbf{B}}^{T} \geq 0
$$

From the Sylvester criterion, one finds that the matrix $\tilde{\mathbf{R}}$ is only a positive semi-definite matrix and therefore we have a defect in the dissipation of the fedback system. From (35), the feedback control law may be given by:

$$
\sum_{i=1}^{m=2} \mathbf{e}_{u_{i, a v}}=-\left(\begin{array}{cc}
\Gamma_{1} & 0 \\
0 & \Gamma_{2}
\end{array}\right)\left(\begin{array}{cc}
\bar{v}_{0}+\bar{v}_{1} & 0 \\
\bar{v}_{0}+\bar{v}_{1} & 0 \\
-\bar{\imath}_{L_{1}}-\bar{\imath}_{L_{2}} & 0 \\
-\bar{\imath}_{L_{1}}-\bar{\imath}_{L_{2}} & -\bar{\imath}_{a} \\
0 & \bar{v}_{0} \\
0 & 0
\end{array}\right)^{T} e
$$

where,

$$
e=\left(\begin{array}{llllll}
e_{L_{1}} & e_{L_{2}} & e_{v_{1}} & e_{v_{0}} & e_{i_{a}} & e_{\omega}
\end{array}\right)^{T}
$$

Note that we use a diagonal matrix $\Gamma=\operatorname{diag}\left(\Gamma_{1}, \Gamma_{2}\right)$, with $\Gamma_{1}$ and $\Gamma_{2}$ being both positive constants. The dissipation matching condition, is not satisfied, but the LaSalle theorem fully applies and it can be seen, with little effort, that the closed loop stabilization error system has a unique equilibrium at the origin and that this equilibrium is asymptotically stable.

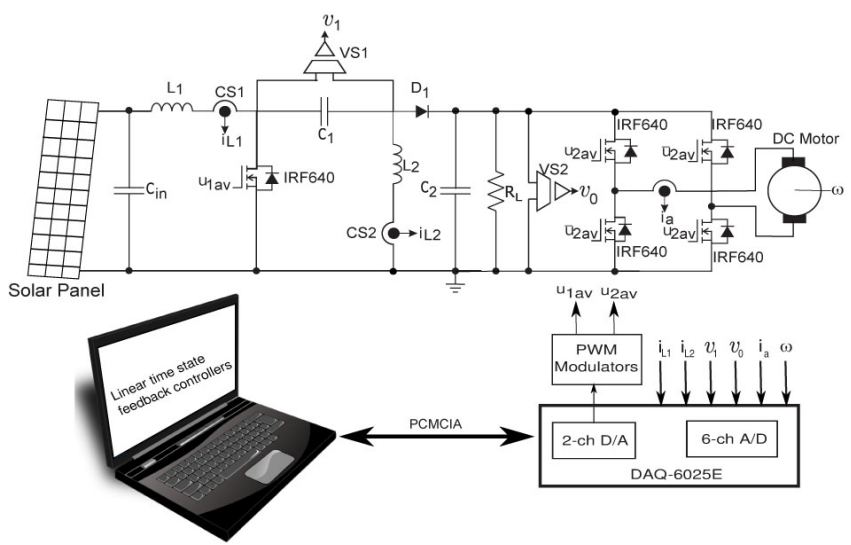

Fig. 2. Experimental setup of the "SFB-converter/dc-motor" system.

The multivariable feedback control law is given by

$$
\begin{aligned}
u_{1, a v} & =\bar{u}_{1, a v}-\Gamma_{1}\left(\bar{v}_{0}+\bar{v}_{1}\right)\left(i_{L_{1}}-\bar{\imath}_{L_{1}}\right) \\
& +\Gamma_{1}\left(\bar{\imath}_{L_{1}}+\bar{\imath}_{L_{2}}\right)\left(v_{1}-\bar{v}_{1}\right) \\
& -\Gamma_{1}\left(\bar{v}_{0}+\bar{v}_{1}\right)\left(i_{L_{2}}-\bar{\imath}_{L_{2}}\right) \\
& +\Gamma_{1}\left(\bar{\imath}_{L_{1}}+\bar{\imath}_{L_{2}}\right)\left(v_{0}-\bar{v}_{0}\right) \\
u_{2, a v} & =\bar{u}_{2, a v}+\Gamma_{2} \bar{\imath}_{a}\left(v_{0}-\bar{v}_{0}\right)-\Gamma_{2} \bar{v}_{0}\left(i_{a}-\bar{\imath}_{a}\right)
\end{aligned}
$$

\section{EXPERIMENTAL SETUP FOR THE SFB-CONVERTER/DC-MOTOR SYSTEM}

The experimental hardware setup is depicted in Fig.2. It is composed of the following devices: a permanent magnet dc-motor of type Hitachi D06D304E with an encoder of 240 pulses/rev, three current sensor circuits; two for the inductors current of the SEPIC power converter and a third one for the armature current of the DC-motor, two voltage sensor circuits; the two sensors are for the voltage measurements of the SEPIC capacitors. The Fig. 2 shows also: an external pulse width modulator circuits $(45 \mathrm{kHz})$, a data acquisition card that serves as a link between the analog circuits and the computer through the Real-Time Windows Target Simulink Library, a computer equipped with MATLAB/Simulink to implement the average controllers.

A data-acquisition card, of type DAQCard-6062E, was used as a hardware interface to MATLAB/Simulink where the algorithm of the linear feedback controllers of the system was programmed. The external PWM-modulators were employed to command the switch positions functions for the power converters. Conventionally, the duty ratio of the PWM is set to the average continuous linear feedback control laws. The PWM-modulators were implemented externally in order not to reduce the transfer capacity of the data acquisition card which can be driven faster than $45 \mathrm{kHz}$.

The design parameters chosen for the SEPIC-Full Bridge converter were: the input voltage of main supply $\bar{v}_{i n}(t)=$ $V_{o p}$ is taken as reference variable in the MPP, which has a performance of rated power $\left(\mathrm{P}_{\max }\right)$ of $50 \mathrm{~W}$ and an optimum voltage in the MPP of $16.8 \mathrm{~V}$ for a solar radiation of $1 \mathrm{KW} / \mathrm{m}^{2}$; The capacitors were set to be: $C_{1}=22 \mu F, C_{2}=470 \mu F$, while the inductors were taken as, $L_{1}=L_{2}=1 \mathrm{mH}$, the resistor was set to be: $R=94 \Omega$. The value capacitor $C_{i n}$ is calculated through (17) with the parameters following: $I_{\text {nom }}=$ 
TABLE I

SPECIFICATIONS OF THE SFB-CONVERTER/DC-MOTOR SYSTEM

\begin{tabular}{|c|c|}
\hline System Parameters & \\
\hline $\mathrm{CS} 1, \mathrm{CS} 2, \mathrm{CS} 3$ & Current sensor (NT-15) \\
\hline VS1 & Voltage sensor (ISO124) \\
\hline VS2 & Voltage sensor (AD620) \\
\hline SEPIC Driver & Single driver (IR2117) \\
\hline Full Bridge Driver & Dual driver (IR2113) \\
\hline Power diode & $D_{1}(\mathrm{BYV} 32)$ \\
\hline Switching device & Mosfet (IRF640) \\
\hline Commutation freq. & $45 \mathrm{Khz}$ \\
\hline Solar panel & SX50U (Solarex) \\
\hline DC Capacitor 1 & $C_{1}=22 \mu F$ \\
\hline DC Capacitor 2 & $C_{2}=470 \mu F$ \\
\hline Input Capacitor & $C_{i n}=2 \mu F$ \\
\hline SEPIC inductors & $L_{1}=L_{2}=1 \mathrm{~m} H$ \\
\hline Resistor load & $R=94 \Omega$ \\
\hline DC-Motor & D06D304E (Hitachi) \\
\hline Armature resitance & $R_{a}=2.0 \Omega$ \\
\hline Armature inductance & $L_{a}=8.9 \mathrm{mH}$ \\
\hline Viscous friction coef. & $B=249.6 \mu \frac{(\mathrm{Nm}-\mathrm{s})}{\mathrm{rad}}$ \\
\hline Moment of inertia & $J=8.2 \mu \mathrm{kgm}^{2}$ \\
\hline Electrical constant & $K_{e}=0.0884 \mathrm{~V} \frac{\mathrm{sec}}{\mathrm{rad}}$ \\
\hline Torque constant & $K_{t}=0.0884 \frac{\mathrm{N}-\mathrm{m}}{\mathrm{A}}$ \\
\hline Sampling Time & $520 \mu \mathrm{sec}$ \\
\hline Optimum voltage & $16.8 \mathrm{~V}$ (MPP) \\
\hline PWM Comparators & LM311 (NS) \\
\hline PWM Generator & ICL8038 \\
\hline
\end{tabular}

$2.97 \mathrm{~A}, V_{\text {nom }}=16.8 \mathrm{~V}, \Delta I=0.048 \mathrm{~A}$, and $\Delta V=4.2 \mathrm{~V}$. Hence, the capacitor value $C_{i n}$ is of $2 \mu F$. The sampling time of the program controller was set to $520 \mu s$. The specifications of the SFB-converter/dc-motor system may be found in Table I.

\section{EXPERIMENTAL RESULTS}

We set constant nominal desired SEPIC output voltage and a nominal desired angular velocity profiles for the SFB converter/DC-motor system. These constant values were specified in accordance with the values given in (20) to (28), hence

$$
\begin{aligned}
& \bar{v}_{0}=\{23 \mathrm{~V} \quad 0 \leq t \leq 10 \\
& \bar{\omega}_{d}= \begin{cases}250 \mathrm{rad} / \mathrm{sec} & 0 \leq t<4 \\
0 & 4 \leq t \leq 6 \\
-250 \mathrm{rad} / \mathrm{sec} & 6<t \leq 10\end{cases}
\end{aligned}
$$

The controller tuning is performed heuristically, where the gamma matrix values are given by the following open intervals:

$$
0<\Gamma_{1}<1 \text { and } 0<\Gamma_{2}<1
$$

When the state variables measurements are equal to zero, each one of the control inputs are equal to the reference variables (pre-feeding). Hence, the multivariable controller operates as a feed-forward control. Once the values of the state variables are different from zero, the control law (38) operates completely and the effects of gamma matrix values are a factor in regulating the error vector. With gamma matrix values near to the value of the unit, the noise level in the system variables is very high, and saturates to controller and causes that the system is been unstable. With positive values near to zero as: $\Gamma_{1}=0.0012$ and $\Gamma_{2}=0.0012$, is reduced the impact of the measurement noise on the system variables and the controller
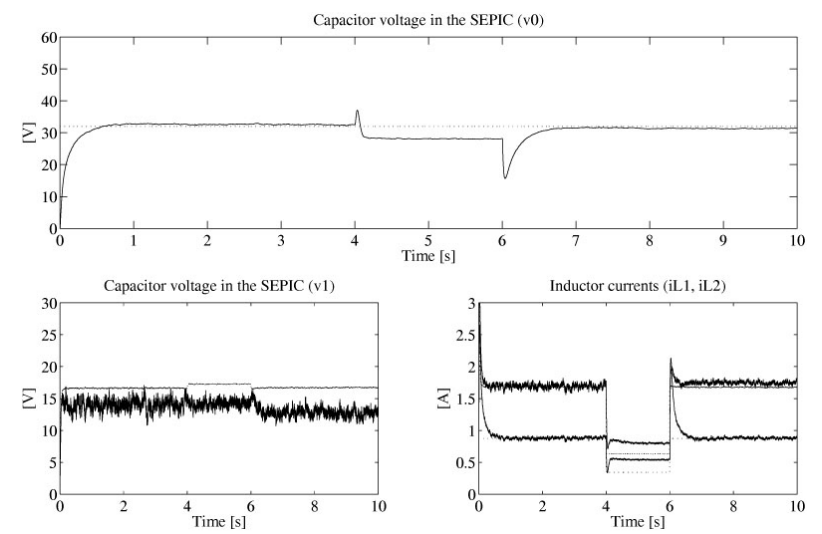

Fig. 3. Experimental results: SEPIC output voltage $v_{0}$ and SEPIC capacitor voltage $v_{1}$, SEPIC currents $i_{L_{1}}$ and $i_{L_{2}}$ for a desired constant output voltage $\bar{v}_{0}$ and a desired constant angular velocity $\bar{\omega}_{d}$ task with no external load torque, $\tau_{L}=0$, reference signals (dashed or dashed-dotted lines), measured signals (solid lines).

is not saturated, and so as to keep the average control signals within its bounds, i.e. $u_{1_{a v}} \in[0,1]$ and $u_{2_{a v}} \in[-1,1]$, while guaranteeing an acceptable regulation of the angular velocity. We measure indirectly the supply of SEPIC converter in the maximum power point (MPP), through equation (1) on steadystate, $\bar{v}_{i n}(t)=\left(1-\bar{u}_{1_{a v}}\right)\left(\bar{v}_{1}+\bar{v}_{0}\right)$, see [21].

Fig. 3 shows the experimental results of the system in closed loop. The graphic in the lower left shows the voltage response for the capacitor $C_{1}$ of the SEPIC converter. In this graph, we see that the voltage on the capacitor $C_{1}$ is slightly lower than the reference voltage in the maximum power point (MPP). The level of noise present is not large in the capacitor voltage $\mathrm{C} 1$ of the SEPIC converter (see Fig. 3), but exist a level of noise due to the transfer energy, i.e., the capacitor $\mathrm{C} 1$ functions as a link element for the transfer energy between the input (power supply) and the output (to the load).

However, the SEPIC output voltage, $v_{0}$, reaches the desired reference value, given by (39). This is the depicted in the upper graphic of Fig. 3. The voltage graphs of the SEPIC converter capacitor shows a decrease in its amplitude in the closed interval, $[4,6] \mathrm{sec}$, while the voltage response in the capacitor $C_{1}$ shows an increase its amplitude slightly, which are produced by a regenerative braking of the motor. The graphic in the lower right shows the responses of the inductor currents.

Fig. 4 shows the experimental results, the upper graphic shows the angular velocity response of the motor shaft for the reference profile given by (40). The average control inputs are shown in the lower left, the control signal, $u_{1, a v}$, shows an average value of approximately 0.68 , while the control signal, $u_{2, a v}$, shows three average values; one for each interval given by the angular velocity reference profile in (40). The first is approximately 0.77 , the second is approximately 0.0 , and third is approximately -0.77 . The armature current response is shown in the lower right. In this graph, current transients are observed, on the armature circuit motor, every time a braking of the motor is performed.

We did other experimental test to check the performance of the controller, this was done in accordance with the values 

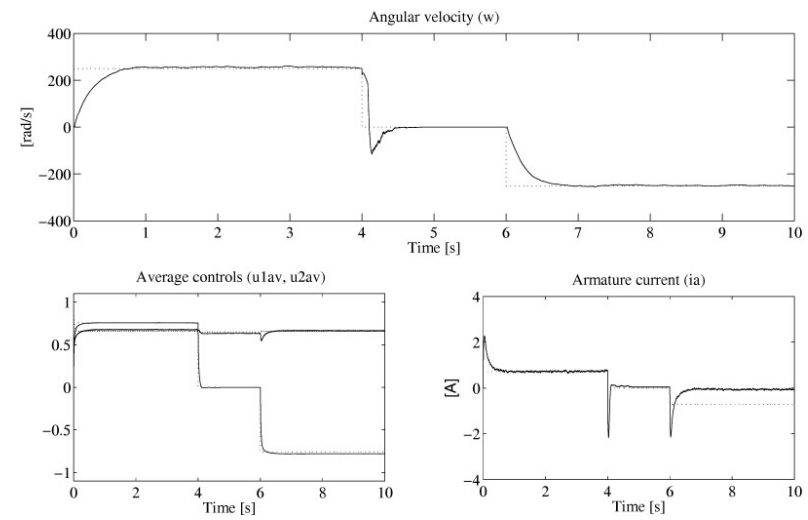

Fig. 4. Experimental results: DC motor angular velocity $\omega$, average controllers $u_{1, a v}, u_{2, a v}$, and armature current $i_{a}$ for a desired constant output voltage $\bar{v}_{0}$ and a desired constant angular velocity $\bar{\omega}_{d}$ task with no external load torque, $\tau_{L}=0$, reference signals (dashed or dashed-dotted lines), measured signals (solid lines).
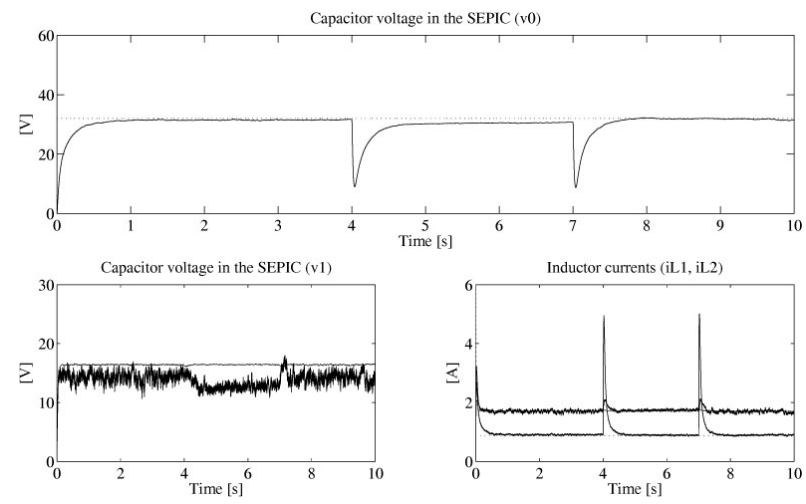

Fig. 5. Experimental results: SEPIC output voltage $v_{0}$ and SEPIC capacitor voltage $v_{1}$, SEPIC currents $i_{L_{1}}$ and $i_{L_{2}}$ for a desired constant output voltage $\bar{v}_{0}$ and a desired constant angular velocity $\bar{\omega}_{d}$ task with no external load torque, $\tau_{L}=0$, reference signals (dashed or dashed-dotted lines), measured signals (solid lines).

given in (20) to (28), hence

$$
\begin{aligned}
& \bar{v}_{0}=\{32 \mathrm{~V} \quad 0 \leq t \leq 10 \\
& \bar{\omega}_{d}= \begin{cases}250 \mathrm{rad} / \mathrm{sec} & 0 \leq t \leq 4 \\
-250 \mathrm{rad} / \mathrm{sec} & 4<t<7 \\
250 \mathrm{rad} / \mathrm{sec} & 7 \leq t \leq 10\end{cases}
\end{aligned}
$$

Fig. 5 and Fig. 6 show the experimental results, where the graphic in the lower left of Fig. 5 shows the voltage response of the capacitor $C_{1}$ of the closed loop SEPIC converter. In this graph, we see that the voltage on the capacitor $C_{1}$ is slightly lower than the optimum voltage $\left(V_{o p}\right)$, but when the desired angular velocity is negative this voltage was decreased a $10 \%$. However, the output voltage response of the SEPIC converter, $v_{0}$, reaches the desired reference value even when the desired angular velocity is negative, given by (42). While the actual inductor currents reach their desired references, these are shown in the lower right of the Fig. 5.

Fig. 6 shows the angular velocity response of the motor shaft for the reference profile given by (42). The average control inputs are shown in the lower left of Fig. 6, the control signal, $u_{1, a v}$, shows an average value of approximately 0.7 , while the control signal, $u_{2, a v}$, shows three average values; one for each interval given by the angular velocity reference profile in (42).

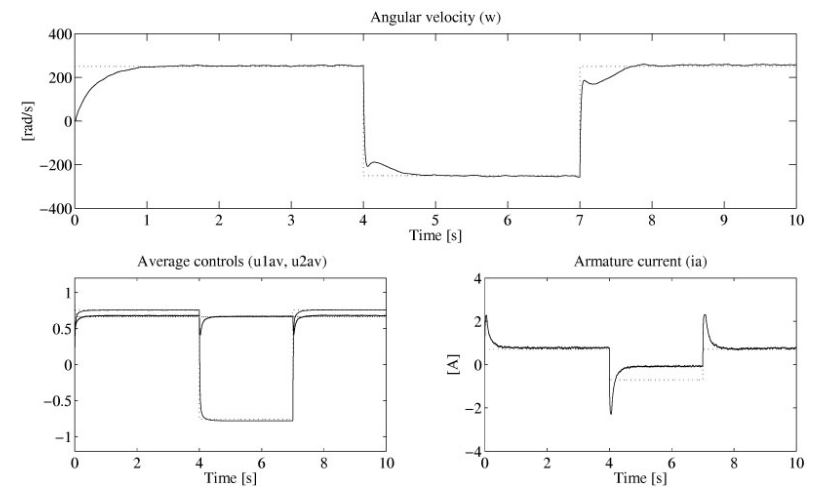

Fig. 6. Experimental results: DC motor angular velocity $\omega$, average controllers $u_{1, a v}, u_{2, a v}$, and armature current $i_{a}$ for a desired constant output voltage $\bar{v}_{0}$ and a desired constant angular velocity $\bar{\omega}_{d}$ task with no external load torque, $\tau_{L}=0$, reference signals (dashed or dashed-dotted lines), measured signals (solid lines).

The first is approximately 0.77 , the second is approximately -0.77 , and third is approximately 0.77 . The armature current response is shown in the lower right of Fig. 6. In this graph, current transients are observed, on the armature circuit motor, every time a braking of the motor is performed.

\section{CONCLUSIONS}

In this article, we have presented a sensor-less controller for a DC motor, driven by a cascade combination of a SEPIC DC/DC converter and a Full Bridge converter, powered via a solar panel. The SEPIC converter exhibits a resistive load and the bridge is loaded by a DC-motor. We simultaneously regulate, both, the output voltage of the SEPIC converter and the angular velocity of the DC-motor shaft by means of switched controlled policies synthesizing the duty ratio functions commanding the switched "actuators" of the cascaded converters in a PWM fashion. The control objectives are 1) to regulate the motor angular velocity to a given constant value in any of the two possible turning senses and 2) to regulate the output voltage of the SEPIC converter to track a constant reference voltage on a known resistive load. This nominal value of the reference voltage is larger than solar panel output voltage in the MPP. The main result of the article is the introduction of a linear time varying passive output feedback controller which is based only on measured variables of the SEPIC converter and on the armature current of the DC motor.

The derived feedback control law is, in fact, a static passivity based controller that uses the exact static error dynamics passive output for feedback purposes. Such a passive output demands the use of electric variables alone. Therefore, no mechanical sensors, such as tacho-meters, are needed for the implementation of the controller. All nominal reference constants demanded by the linear feedback controller are generated through the equilibrium constant points of the combined multi-variable system. The controller reference variables depend of the solar panel output voltage. For this reason, we set these in terms of the solar panel output voltage at the MPP value.

The discrete switching control realization of the designed average continuous feedback control law is accomplished by 
means of a PWM-modulation scheme. In this article, we present actual experimental results depicting the closed loop performance of the system under the proposed multi-variable linear time varying controller. Further experimental studies are possible and other challenging problems remain to be solved. Suitable modifications of the proposed controller to achieve robustness with respect to resistive load variations, solar panel output voltage variations, and unforseen load torques affecting the motor shaft are being pursued at the present time. These entitle adaptive feedback control schemes and also algebraic, on-line, parameter estimation options.

\section{ACKNOWLEDGMENT}

The authors wish to thank the anonymous reviewers for their helpful comments and suggestions. This work was supported by the "Programa de Mejoramiento del Profesorado" under grant number PROMEP/103.5/10/4984 and folio assigned to the professor UTMIX-PTC-020.

\section{REFERENCES}

[1] E. Mineiro, S. Daher, F. L.M. Antunes, and C. M. T. Cruz, "Photovoltaic system for supply public illumination in electrical energy demand peak," in Proceeding of Applied Power Electronics Conference and Exposition (APEC), Vol. 3, pp. 1501-1506, 2004.

[2] L. Egiziano, A. Giustiniani, G. Lisi, G. Petrone, G. Spagnuolo, and M. Vitelli, "Experimental characterization of the photovoltaic generator for a hybrid solar vehicle," in Proceeding of IEEE ISIE, pp. 329-334, 2007.

[3] T. Friedli, S. D. Round, and J. W. Kolar, "Modeling the space elevator-A project oriented approach for teaching experimental power electronics," in Proceeding of European Conference on Power Electronics and Applications, pp. 1-10, 2007.

[4] J. R. Higinbotham, J. R. Moisan, C. Schirtzinger, M. Linkswiler, J. Yungel, and P. Orton, "Update on the development and testing of a new long duration solar powered autonomous surface vehicle," in Proceeding of Oceans, pp. 1-10, 2008.

[5] E. E. Jiménez-Toribio, A. A. Labour-Castro, F. Muñiz-Rodríguez, H. R. Pérez-Hernández, and E. I. Ortiz-Rivera, "Sensorless sontrol of SEPIC and cuk converters for DC motors using solar panels," in Proceeding of IEEE International Electric Machines and Drives Conference (IEMDC), pp. 1503-1510, 2009.

[6] J. Linares-Flores and H. Sira-Ramírez, "DC motor velocity control through a DC-to-DC power converter," in Proceeding of 43rd IEEE Conference on Decision and Control, pp. 5297-5302, 2004.

[7] J. Chiasson, Modeling and High-Performance Control of Electric Machines, John Wiley, New York, 2005.

[8] H. Fadil, F. Giri, F. Z. Chaoui, and O. El Magueri, "Accounting for input limitation in the control of buck power converters," IEEE Trans. Circuits Syst. I, Reg. Papers, Vol. 56, No. 6, pp. 1260-1271, Jun. 2009.

[9] J. Linares-Flores, J. Reger, and H. Sira-Ramírez, "Load torque estimation and passivity-based control of a boost-converter/DC-motor combination," IEEE Trans. Contr. Syst. Technol., Vol. 18, No. 6, pp. 1398-1405, Nov. 2010.

[10] J. Linares-Flores, J. Reger, and H. Sira-Ramírez, "A time-varying linear state feedback tracking controller for a Boost-converter driven DC machine," in Proceeding of 4th IFAC-Symp. Mechatron. Syst., Heidelberg, Germany, 2006.

[11] J. Linares-Flores, J. Reger, and H. Sira-Ramírez, "Speed-sensorless tracking control of a DC-motor via a double Buck-converter," in Proceeding of 45th IEEE Conference on Decision and Control, pp. 62296234, 2006.

[12] M. M. R. Ahmed and G. A. Putrus, "Fuzzy logic speed control of D.C. motors fed by single-ended primary inductance convertes (SEPIC)," in Proceeding of the 41st Universities Power Engineering Conference (UPEC), pp. 343-347, 2006.

[13] H.-E. Park and J.-H. Song, "A dP/dV feedback-controlled MPPT method for photovoltaic power system using II-SEPIC," Journal of Power Electronics, Vol. 9, No. 4, pp. 604-611, Jul. 2009.
[14] H. Shu-Hung Chung, K. K. Tse, S. Y. Ron Hui, C. M. Mok, and M. T. Ho, "A novel maximum power point tracking technique for solar panels using a SEPIC or cuk converter," IEEE Trans. Power Electron., Vol. 18, No. 3, pp. 717-724, May 2003.

[15] S. J. Chiang, H.-J. Shieh, and M.-C. Chen, "Modeling and control of PV charger system with SEPIC converter," IEEE Trans. Ind. Electron., Vol. 56, No. 11, pp. 4344-4353, Nov. 2009.

[16] H. Sira-Ramírez and R. Silva-Ortigoza, Control Design Techniques in Power Electronics Devices, Power Systems Series, Springer-Verlag, London, U.K., 2006.

[17] E. I. Ortiz Rivera and F. Z. Peng, "Linear reoriented coordinates method," in Proceeding of IEEE Internacional Conference on Electro/Information Technology, pp. 459-464, 2006.

[18] L. Fangrui, D. Shanxu, L. Fei, L. Bangyin, and K. Yong, "A variable Step Size INC MPPT Method for PV Systems," IEEE Trans. Ind. Electron., Vol. 55, No. 7, pp. 2622-2628, Jul. 2008.

[19] SX50U data sheet-http://www.oksolar.com/panels/solarex.html, August 13th 2010

[20] H. Khalil, Nonlinear Systems, Third Edition, Prentice-Hall, NJ, 2002.

[21] H. Y. Kanaan and K. Al-Haddad, "A novel averaged-model-based control of a SEPIC power factor corrector using the input/output feedback linearization technique," in Proceeding of IEEE 36th Power Electronics Specialists Conference, PESC '05, pp. 565-571, 2005.

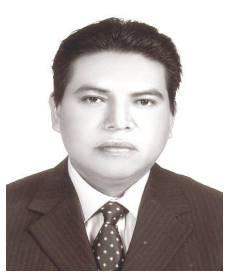

Jesús Linares-Flores received the bachelor's degree in Electronics Engineering from Universidad Autónoma de Puebla in 1994, the MSc. degree from Universidad de las Américas-Puebla in 1999, and the Ph.D. degree from Centro de Investigación y de Estudios Avanzados del I.P.N. in 2006. Since 2007, he is representative of the academic corp with name Automatization and Control of the mechatronics systems UTMIX-CA-24PROMEP. He is Director of the Institute Electronics and Mechatronics at Universidad Tecnológica de la Mixteca. He is author of 20 technical papers in credited journals and international conferences. $\mathrm{He}$ is a SNI Member of the CONACYT-MEX. Dr. Linares-Flores is interested in the theoretical and practical aspects of feedback regulation of linear and nonlinear dynamic systems with special emphasis in Passivity-Based Control techniques and its applications in Power Electronics.

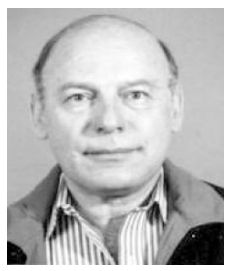

Hebertt Sira-Ramírez obtained the Electrical Engineers degree from the Universidad de Los Andes in Mérida (Venezuela) in 1970. He later obtained the MSc in $\mathrm{EE}$ and the Electrical Engineer degree, in 1974, and the $\mathrm{PhD}$ degree, also in $\mathrm{EE}$, in 1977, all from the Massachusetts Institute of Technology (Cambridge, USA). Dr Sira-Ramírez is a Senior Member of the Institute of Electrical and Electronics Engineers (IEEE), a Distinguished Lecturer from the same Institute and a Member of the IEEE International Committee. He is a coauthor of several books on Automatic Control and the author of over 400 technical papers in credited journals and international conferences. Dr. Sira-Ramírez is interested in the theoretical and practical aspects of feedback regulation of nonlinear dynamic systems with special emphasis in Variable Structure feedback control techniques and its applications in Power Electronics.

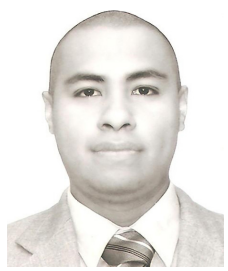

Edel F. Cuevas-López was born in Oaxaca de Juárez, México in 1982. He received a bachelor's degree in Electronics in 2008 at Universidad Tecnológica de la Mixteca, Huajuapan, México. He is currently a M. Sc. candidate in Electronics at Universidad Tecnológica de la Mixteca, Huajuapan, México. His research interest is in the DC-DC converters applied to motor control.

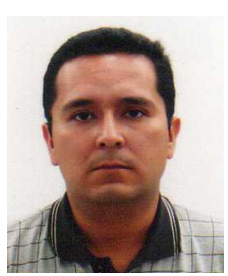

Marco A. Contreras-Ordaz was born in Matías Romero, México, in 1974. He received a M. Sc. degree in Electronics in 2001 and his Ph. D. degree in Electronics in 2007 at Centro Nacional de Investigación y Desarrollo Tecnológico, Cuernavaca, México. He is currently a Professor for the Instituto de Electrónica y Mecatrónica at Universidad Tecnológica de la Mixteca, Huajuapan, México. His current research interests include power quality, multilevel converters and integration of renewable energy. 